\title{
Erratum: Extended Falicov-Kimball model: Exact solution for finite temperatures [Phys. Rev. B 99, 245143 (2019)]
}

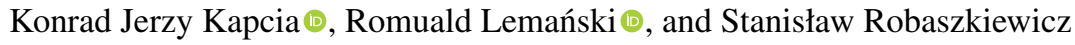

(Received 1 June 2020; published 23 June 2020)

DOI: 10.1103/PhysRevB.101.239901

Equation (16) in the original paper does not contain the factor of $\left(-k_{B} T\right)$ before the integral. This is a purely typographical error. However, let us note that free energy $F=F_{\text {el }}+F_{\text {ions }}+U / 4+V$ of the extended Falicov-Kimball model calculated using corrected Eqs. (16) and (17) has a fundamental meaning because only after its minimization with respect to order parameter $d$ we obtain $d(T)$ and $d_{1}(T)$ that enter into all physical characteristics of the system (in the approach used), which are calculated in the original paper. Thus, it is extremely important to use the correct equation for $F_{\mathrm{el}}$ given as follows:

$$
F_{\mathrm{el}}=-k_{B} T \int_{-\infty}^{\infty}\left\{\rho\left(U, V, d, d_{1} ; \varepsilon\right) \ln \left[1+\exp \left(-\frac{\varepsilon}{k_{B} T}\right)\right]\right\} d \varepsilon-V\left(1-d_{1}^{2}\right) / 4
$$

where $\rho\left(U, V, d, d_{1} ; \varepsilon\right)=\left[\rho^{+}(\varepsilon)+\rho^{-}(\varepsilon)\right] / 2$ and $\rho^{ \pm}(\varepsilon) \equiv \rho^{ \pm}\left(U, V, d, d_{1} ; \varepsilon\right)$ are expressed by Eq. (8) of the original paper (they are non-negative by definition), $d$ and $d_{1}$ are calculated from Eqs. (4) and (9) of the original paper.

Moreover, the integration in Eq. (9) of the original paper is done in the range of energies $\varepsilon$ from $-\infty$ to $+\infty$ (over all energies), not only over energies below the Fermi level located at $\varepsilon_{F}$ as it was given initially in the original paper. Thus, the correct form of Eq. (9) for concentrations $n_{\downarrow}^{ \pm}$of electrons with spin- $\downarrow$ is

$$
n_{\downarrow}^{ \pm}=\int_{-\infty}^{+\infty} \frac{\rho^{ \pm}\left(U, V, d, d_{1} ; \varepsilon\right)}{1+\exp \left[\varepsilon /\left(k_{B} T\right)\right]} d \varepsilon
$$

Let us also clarify that the Fermi level $\varepsilon_{F}$ at finite temperatures $(T>0)$ in this work should be understood as the chemical potential $\mu=U / 2+2 V$, which is located at $\varepsilon=0$, in particular, in Figs. 1 and 2 as well as in Secs. III A 1 and III A 2 of the original paper.

We should underline that in all analytic derivations and all numerical analysis presented in the original paper, we used the correct expressions mentioned above. Thus, these corrections do not affect any other part of the paper, in particular, any other equations, the results, the discussion, and the conclusions included in the original paper.

We thank Jakub Krawczyk for bringing these errors to our attention. 